\title{
Video Article \\ Evaluation of Patients' Posture and Gait Profile After Lumbar Fusion Surgery by Video Rasterstereography and Treadmill Gait Analysis
}

\author{
Sebastian Scheidt ${ }^{1,2}$, Ulf Krister Hofmann ${ }^{2}$, Falk Mittag ${ }^{2}$ \\ ${ }^{1}$ Department of Orthopedics and Trauma Surgery, University Hospital Bonn \\ ${ }^{2}$ Department of Orthopedic Surgery, University Hospital Tuebingen
}

Correspondence to: Sebastian Scheidt at sebastian.scheidt@outlook.com

URL: https://www.jove.com/video/59103

DOI: doi: $10.3791 / 59103$

Keywords: Behavior, Issue 145, video rasterstereography, treadmill, gait analysis, lumbar back pain, posture, sagittal balance, TLIF, spine surgery, lumbar fusion surgery

Date Published: $3 / 23 / 2019$

Citation: Scheidt, S., Hofmann, U.K., Mittag, F. Evaluation of Patients' Posture and Gait Profile After Lumbar Fusion Surgery by Video Rasterstereography and Treadmill Gait Analysis. J. Vis. Exp. (145), e59103, doi:10.3791/59103 (2019).

\section{Abstract}

This protocol provides guidance on how to perform high resolution video rasterstereography and treadmill gait analysis on patients after lumbar fusion surgery to obtain results about altered variables of gait and posture. These observed changes can then be correlated with the patientreported outcome measure of pain relief. The rasterstereographic device projects lines of parallel light onto the surface of the tested subject's back. The deformation of these lines is recognized by the device. From these data, a special software then generates a 3-D profile based on the principle of triangulation. With an inaccuracy of only $0.2 \mathrm{~mm}$ it can measure changes in posture at very high precision. Gait and stance parameters are recorded using a treadmill equipped with an electric sensor mat that contains 10,200 miniature force sensors in the registering zone under the belt. Initial walking speed on the treadmill is $0.5 \mathrm{~km} / \mathrm{h}$. Speed is then gradually increased by increments of $0.1 \mathrm{~km} / \mathrm{h}$ until each subject reaches his or her individual maximum well tolerable walking speed. At this speed, parameters are recorded during a $20 \mathrm{~s}$ measurement interval. Subjects are tested barefoot and without holding a handrail. Among various other parameters, stride width, step length, stance phase and foot rotation are measured. Both methods used reportedly have a high intra- and inter-observer reliability. The advantage of these highly accurate techniques is that they offer an objective and very detailed perspective on changes in the patient's posture and gait. Due to the amount of data generated, these techniques are, however, not so much suitable for everyday routine use, but rather interesting to scientifically evaluate long term alterations in posture and gait in patients like for example after lumbar fusion surgery.

\section{Video Link}

The video component of this article can be found at https://www.jove.com/video/59103/

\section{Introduction}

This protocol provides instructions on how to objectively perform a functional posture and gait analysis of patients after lumbar spinal fusion surgery in contrast to subjective evaluation by the examiner or patient reported questionnaires. The setup consists of a high-resolution video rasterstereography for posture analysis, and a pressure-sensor equipped treadmill setup for gait analysis. Results obtained by these techniques from patients after lumbar fusion surgery are compared with subjectively reported pain relief.

Even if spinal surgery techniques and outcomes have vastly improved over the past years, the increase in procedures performed ${ }^{1,2}$ also leads to a rise in absolute numbers of patients dissatisfied with their individual postoperative results. For surgeons, it is thus crucial to identify those patients who will most likely benefit from surgery. The development of this skill is closely associated with the constant postoperative outcome evaluation and reevaluation of the initial indication for surgery.

To date, the postoperative outcome is mostly judged on subjective patient-reported levels of pain and function by questionnaires ${ }^{3,4,5}$. These questionnaires are, however, always subjectively affected and not only influenced by the objective physical abnormality but also by the patient's attitudes and beliefs, psychological distress, and illness behavior. Interestingly, even findings in X-ray, computed tomography or magnetic resonance imaging are prone to high inter- and intra-observer variability $6,7,8,9,10$. The additionally radiologic imaging, however, only offers a static technical evaluation of the surgery. There is a clear lack in means to objectively evaluate the functional outcome after spinal surgery.

A patient's posture and gait are generally supposed to be linked to the perceived level of pain and also to the overall quality of life ${ }^{11,12}$. Therefore, function can be considered one of the most important elements of postoperative outcome. The overall functional satisfaction of the patient seems to be associated with spinal alignment, kyphosis, lordosis and vertebral rotation ${ }^{13,14,15}$. As lumbar fusion surgery tries to restore the anatomical curvature of the spine and therefore to balance the muscles, the adaptation of posture is expected ${ }^{16}$. Restored lumbar lordosis is complementary with pain relief and thus result in the ability to walk painless.

The technique of back surface analysis goes back to the work of Takasaki and Meadows et al., as well as Drerup et al. from the late 1970s and $80 \mathrm{~s}^{17,18,19,20,21}$. Based on the principle of triangulation, this technique presents a measurement inaccuracy of only $0.2 \mathrm{~mm}{ }^{22}$. The technique 
is widely used and tested for the radiation free diagnosis and follow-up of patient with scoliosis ${ }^{23,24}$. In the context of evaluation of scoliosis patients, the setup showed good validity and an excellent intra- and interrater reliability ${ }^{25}$. An even more functional view on the patient offers the analysis of gait. A common technique to register the distinct parameters used to describe a patient's gait is a treadmill experimental setup. Thus stride width, step length, stance phase and foot rotation as well as pressure distribution for each foot can be measured at a very high precision ${ }^{26,27,28,29,30,31}$. Whereas patients with low back pain seem to use strategies to reduce the impact on the lumbar spine while walking, the treadmill setup offers the advantage to measure a patient's walk while keeping track of every single step $^{32}$.

The hypothesis is that lumbar fusion surgery changes pathologic patterns in gait or posture and that these changes are in correlation with the detectable alleviation in the patient-reported outcome measure i.e., level of pain. The expected changes can be measured with video rasterstereography and treadmill gait analysis. The additional information about posture and gait can thus be compared with the overall functional status and satisfaction ${ }^{14,15,33}$.

\section{Protocol}

Full approvals from the Department of Orthopaedic Surgery at the University of Tuebingen and the Ethics Committee at the University Hospital Tuebingen were obtained before the commencement of the study. Written informed consent was received from all subjects before their participation.

\section{Patient Recruiting and Preparation}

1. Recruit a subject, aged more than $\mathbf{1 8}$ years, who suffers from lumbar back pain and degenerative disc disease.

1. Gather all relevant data as back pain related patient history, results from magnetic resonance imaging, current pain medication and history of physiotherapy.

2. Perform an orthopedic physical examination to identify the origin of the lumbar back pain looking for tender pressure points, test lateral flexion and trunk inclination and extension, and perform the straight leg raise. For differential diagnosis also test the hip joint for example for flexion, extension and rotation.

NOTE: 30 subjects and 28 reference subjects were used for the original study.

2. Rule out that the subject has a neurologic deficit of the lower limbs that requires immediate surgery by physical examination of each of the key muscles.

NOTE: A deficit in the sensorimotor system of the lower limb of less than grade $3 / 5$ (Janda's classification) should not be included in this study.

3. Ensure that the subject presents with normal walking ability and does not show any acute neoplastic or infectious pathology of the spine. NOTE: The neoplastic or infectious pathology of the spine will be visible in the magnetic resonance imaging.

4. Schedule the subject for spinal surgery.

5. Ask all subjects to sign an informed consent for participating in the study.

6. Schedule measurement dates for the following experimental setup (see 1.7, 1.8, 1.9., 1.10.) with the subject.

7. Perform the first measurement one day prior to surgery.

8. Perform the second measurement approximately seven days after surgery, when walking on ward-level is regained.

9. Schedule and perform the third measurement three-months postoperatively.

10. Schedule and perform the fourth measurement one-year postoperatively.

NOTE: During each examination ask the subject to complete the Oswestry Disability Index (ODI) ${ }^{34}$ questionnaire and to indicate their usual value on the Numeric Pain Rating Scale (NRS) ${ }^{35}$.

11. Perform the gait and posture analysis with the subject on each visit following the subsequent instructions under section 2 of the protocol.

\section{Experimental Design}

1. Questionnaires

1. Ask the subject to complete the Oswestry Disability Index (ODI) questionnaire and to indicate his or her usual value on the Numeric Pain Rating Scale (NRS).

2. Rasterstereographic analysis

1. Implement the measurement setup.

1. Use a device based on the principle of optical stereographic measuring that allows the detection of the specific anatomical landmark's vertebra prominens, the two lumbar dimples, and the sacrum point of the rima ani.

2. Use an apparatus that estimates spine configuration on the Moiré principle using a projector that projects a grid of light lines on the patient's back and contains a light-optical scanning camera.

NOTE: Based on the principles of triangulation, the software analyzes the projected lines and generates a 3-D model of the patients' surface $(7,500$ points).

3. Build the measurement system with two main modules: the light projector unit that emits the projections of parallel lines and captures the reflections with a camera $(15 \mathrm{~Hz})$ and a personal computer with the manufacturer's analysis-software installed.

4. Additionally, hang up a $2.5 \mathrm{~m} \times 2 \mathrm{~m}$ piece of plain black cloth or similar that entirely covers the background of the image taken to improve the contrast.

2. Begin the measurement-process by asking the subject to undress from head down to the waist to expose all four needed anatomical landmarks: the neck with the vertebra prominens, the two lumbar dimples, and the sacrum point as the cranial end of the rima ani.

3. Make sure that especially the caudal landmarks are also visible. This may require that the subject opens the trousers and lowers them a little. 
4. Let the subject stand freely and barefoot in a relaxed standard anatomical position with the feet shoulder-wide apart.

5. Position the subject's front facing towards the wall with the black background while his or her back is targeted to the camera device.

1. Measure the distance from the subject's back surface to the camera device with a measuring tape, as it needs to be at $200 \mathrm{~cm}$ during all measurements.

6. Begin the measurement by clicking the button for the software's automatic landmark detection on screen while the subject stands freely, barefoot in a relaxed standard anatomical position with the feet shoulder-wide apart.

1. In case of a scanning error manually re-adjust the landmarks position according to the manufacturer's instructions provided with the software, so that they match their actual anatomic position (see step 2.2.2).

7. Set the system to a measurement time of $30 \mathrm{~s}$. Due to the $15 \mathrm{~Hz}$ rate of the camera device about 450 images will be captured.

8. Click Generate on the software panel and wait for the results. The software will calculate the average terminal values needed for further analysis.

9. Let the subject rest for $120 \mathrm{~s}$ and subsequently step on the treadmill device.

\section{Treadmill Gait Analysis and (Optional) Plantar Pressure Measurements}

1. Use an instrumented treadmill with an integrated system containing capacitive pressure sensors under the belt to register gait parameters such as stride width, step length, stance phase and foot rotation.

1. Make sure to use a measuring system that contains of 10,200 miniature $0.85 \mathrm{~cm} \times 0.85 \mathrm{~cm}$ capacitive pressure sensors on a mat of $150 \mathrm{~cm} \times 50 \mathrm{~cm}$, registering the exerted force at a rate of $120 \mathrm{~Hz}$ and which has a spatial resolution of the mat of $1.4 \mathrm{sensors} / \mathrm{cm}^{2}$.

2. At first, connect the treadmill and video camera to a commercial personal computer using the manufacturer's measurement software.

3. Ask the subject to stand on the treadmill barefoot and with the pants rolled up to the knees.

4. Attach a safety plug to the subject's shirt.

NOTE: The safety belt ensures measurement safety by an automatic shutdown of the treadmill, if the subject stumbles or is pushed too far back by the belt. In addition, the treadmill can be shut off via an emergency stop button or a cord.

5. Use two lateral rail bars attached to the sides of the treadmill, to prevent the patient from falling off the treadmill in case of stumbling.

6. Set the slope of the treadmill at $0 \%$ during the entire measurement.

NOTE: If necessary, the slope of the treadmill used in this study can be adjusted in a range from $-2 \%$ to $+15 \%$ in $0.5 \%$ increments, to simulate up-hill walking.

7. To register the total load distribution on each foot, ask the subject to stand freely on the treadmill sensors thrice for $10 \mathrm{~s}$. Then calculate the mean value of these three measurements.

8. In the next step, when the treadmill is turned on, ask the subject to walk with normal gait and, as far as possible, not to hold on to the handrails.

NOTE: Walking on the treadmill without holding the handrail is recommended to obtain more dependable results and achieve higher reliability.

9. Furthermore, advise the subject to walk between two adhesive tape markers you accurately attached beforehand on the surface of the treadmill to define the limits of the integrated sensor mat.

10. After starting the treadmill, increase the speed in small increments of $0.1 \mathrm{~km} / \mathrm{h}$ starting from $0.5 \mathrm{~km} / \mathrm{h}$ until the subject's individual maximum well tolerable walking speed is reached. Ask the subject during the increase how he or she feels comfortable walking.

NOTE: The maximum well tolerable walking speed is reached when the subject has reached the highest walking speed with which he or she feels still comfortable walking. The belt speed can be upregulated in $0.1 \mathrm{~km} / \mathrm{h}$ increments to a maximum speed of $22 \mathrm{~km} / \mathrm{h}$ which thus even allows running measurements. The minimum speed of the treadmill is $0.5 \mathrm{~km} / \mathrm{h}$.

11. For every subject measure two trials with a duration of $20 \mathrm{~s}$. Let the subject rest for $60 \mathrm{~s}$ between the trials. NOTE: The trial speed is specified by the individual walking speed determined in step 3.10.

12. Film the subject's gait at the same time with a video camera from behind to allow visual correlation between the actual gait profile and the assessed parameters.

13. Print the results displayed as a report through the software's interface at the end of the measurement.

NOTE: To further quantify foot pressure distribution during gait, the development of a software tool to subdivide the foot into different regions is necessary. For each region of interest pressures are registered from heel strike to toe-off during each gait cycle in N/cm². Eight distinct regions are defined: hindfoot, midfoot, first metatarsal head, second/third metatarsal head, fourth/fifth metatarsal head, hallux, second/third toe and fourth/fifth toe.

\section{Experimental Design - Statistical Analysis}

1. Analyze the data obtained in step 2.2.8 and 3.13 using commercially available statistical software (Table of Materials). Import the data to the software by clicking Import.

1. Assess normality for the data obtained in step 2.2 .8 and 3.13 by using histograms, the Shapiro-Wilk or Kolmogorov-Smirnoff test depending on the sample-size, and equality of variances by using the Levene test.

2. Present data as mean (standard deviation) or median (minimum-maximum), depending on normality.

3. Present categorical variables as relative or absolute frequencies.

4. For treadmill variables organize each patient's bilateral data into major and minor values and calculate their absolute differences as a parameter for gait symmetry.

5. For demographic characteristics use the Kruskal-Wallis test, chi-squared test, Friedman test, Wilcoxon test, and Tukey test, depending on normality.

6. Calculate correlations between the measurement changes and changes in the patient-reported outcome measures between different time-points using Kendall's tau. 
7. Calculate NRS values as a percent of the initial value.

1. When grouping improvement on the Numeric Pain Rating Scale (NRS) ordinally, consider $>75 \%$ an excellent, $30-74 \%$ a moderate, and $<30 \%$ as no improvement.

NOTE: Since it is impossible to distinguish those patients with actual pain improvement $<30 \%$ accompanied by also functional improvement from those with improvement just due to a placebo effect (which can reach up to $30 \%$ improvement) where we would not expect functional changes, we classified this group for study purposes as "no improvement" ${ }^{55,36}$

8. Interpret the Oswestry Disability Index (ODI) according the questionnaire's instructions.

1. Interpretation of the ODI: For each section, the total possible score is five. After all of the ten sections are completed by the patient, calculate the score as follows. Divide the selected total score by the total possible score (50) multiplied by 100 to obtain the final score in percent. For each section that is missed or not applicable the total score by which to divide is lowered by five. Interpretation of the final score: 0-20\%: minimal disability, $21-40 \%$ : moderate disability, $41-60 \%$ : severe disability, $61-80 \%$ : crippled, $81-100 \%$ : exaggerating patient or bed-bound

\section{Representative Results}

The representative results shown in this protocol come from a previous publication that has been published elsewhere ${ }^{26}$.

\section{Rasterstereographic analysis}

The results of perioperative rasterstereographic analysis of patients who did suffer from chronic lumbar back pain and who were treated with lumbar fusion surgery $(n=59)$ showed no significant changes in trunk length at the 3 month follow-up in comparison to the preoperative measurements (459 (33) - $448(40) \mathrm{mm} ; \mathrm{p}=0.313$; Tukey test) (Figure 1A). We however noted a significantly reduced kyphotic angle (vertebra prominens (VP) - thoracic spine vertebrae 12 (Th12), from $52^{\circ}$ to $43^{\circ} ; p=0.014$; Tukey test) and lordotic angle (Th12 - dimple medium (DM), from $28^{\circ}$ to $11^{\circ} ; p<0.001$; Tukey test) at the first post-operative measurement when compared to the preoperative values (Figure 1B). No differences for the measurements of trunk inclination or lateral tilt were detected at any time point (Figure 1C, D).

\section{Gait and stance analysis}

The treadmill gait measurements of the same patient cohort $(n=59)$ showed a significant reduction in cadence in the course from preoperatively to 3 months postoperatively (pre-OP to 7-days postoperatively: 98 (57-132) - 94 (43-119) steps/minute, $p=0.004$; 3-months postoperatively: 91 (54-117) steps/minute, $p=0.006$, Wilcoxon-test) (Figure 2A). Over the three postoperative months significant changes were detected for most spatiotemporal parameters (swing phase $p=0.01$; stance phase $p<0.001$; foot rotation $p=0.001$ ). However, no significant improvements were seen for the symmetry of swing phase (difference-major-minor value (DiffMJMn) $2(0-8)-1(0-6) \%)$, stance phase (DiffMJMn 2 (0-8) - $1(0-6) \%$ ) or foot rotation (DiffMJMn $3(0-10)$ - $3(0-15)^{\circ}$ ) (Figure 2B,C,D).
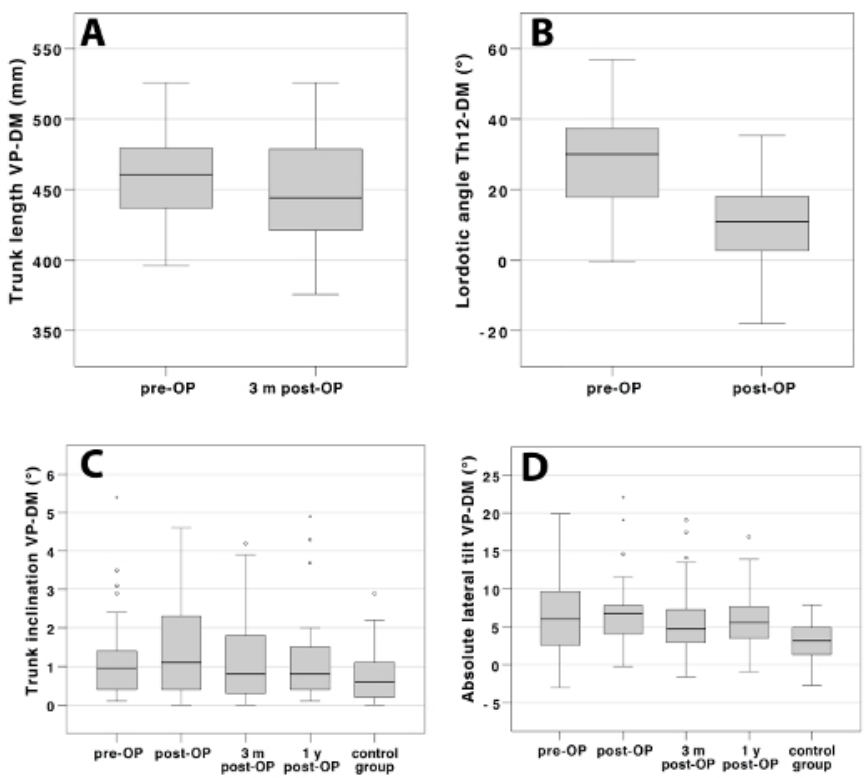

Figure 1: Rasterstereographic results. Boxplots displaying measurement changes for $(\mathbf{A})$ trunk length at the 3-months follow-up in comparison to the preoperative measurements (459 (33) - $448(40) \mathrm{mm} ; \mathrm{p}=0.313$; Tukey test), (B) Lordotic angle at the first postoperative measurement when compared to the preoperative values (thoracic spine vertebra 12 - dimple medium, from $28^{\circ}$ to $11^{\circ} ; p<0.001$; Tukey test), and (C-D) trunk inclination and lateral lilt over the course of one year (no significant difference). This figure has been adapted from reference ${ }^{26}$. Please click here to view a larger version of this figure. 

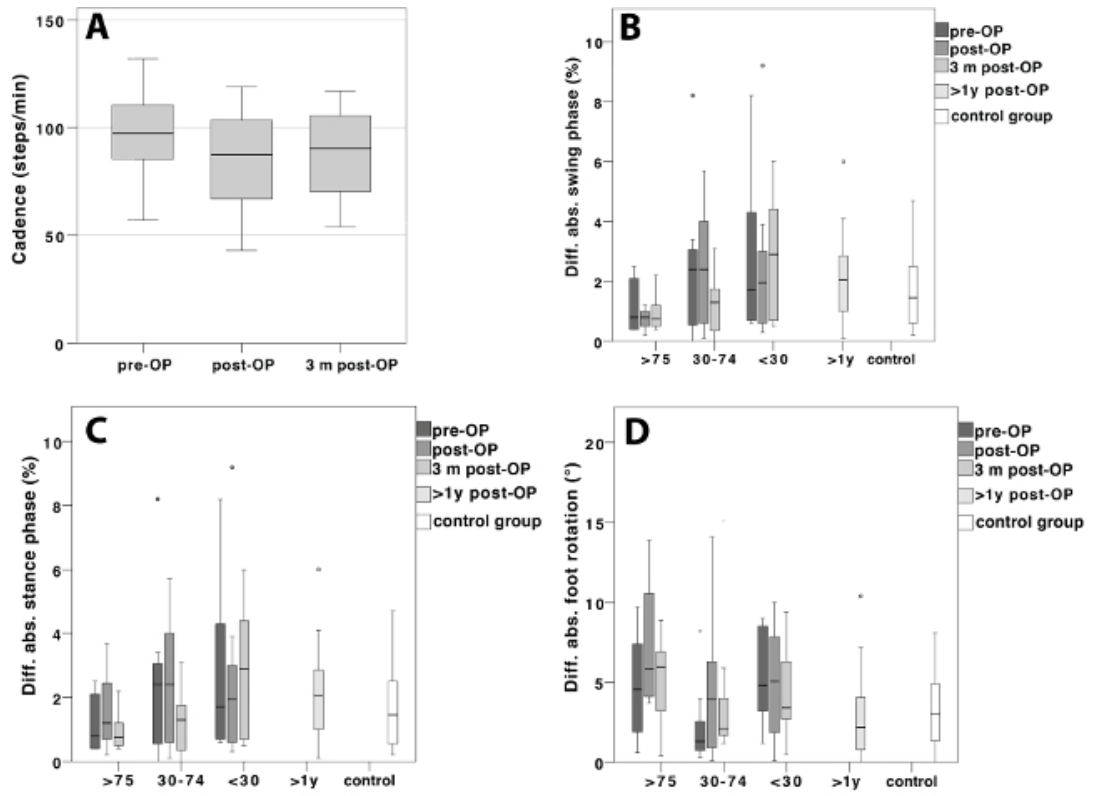

Figure 2: Gait and stance results. (A) Boxplots displaying a reduction in cadence from preoperatively over the postoperative course of 3 months (preoperatively to 7 days postoperatively: 98 (57-132) - 94 (43-119) steps/minute, $p=0.004 ; 3$ months postoperatively: 91 (54-117) steps/minute, $p=0.006$, Wilcoxon-test) and the preoperative, 7-days and 3-months postoperative treadmill results for (B) swing phase, (C) stance-phase and (D) foot-rotation grouped according to subjective pain relief after surgery in percentage $(<30 \%, 30-74 \%,>75 \%)$. From preoperatively to 3-months postoperatively we detected significant changes for most spatiotemporal parameters (swing phase $p=0.01 ;$ stance phase $p<0.001$; foot rotation $p=0.001)$. No significant improvements were however observed with respect to their effect on gait symmetry (swing phase (difference-major-minor value (DiffMJMn) $2(0-8)$ - $1(0-6) \%)$ stance phase (DiffMJMn $2(0-8)$ - $1(0-6) \%$, ), or foot rotation $\left(\right.$ DiffMJMn $\left.3(0-10)-3(0-15)^{\circ}\right)$ ). This figure has been adapted from reference ${ }^{26}$. Please click here to view a larger version of this figure.

\section{Discussion}

Perioperative surgical outcome-monitoring is a field that is subjectively shaped. First it is affected by the surgeon's experience and secondly by the patient's subjective perception registered by for example questionnaires which also reflect his or her psychological distress and illness behavior. Our presented procedure offers an approach that objectifies crucial parameters regarding functional outcome. The methodical setup presented in this manuscript allows high precision measurements of changes in posture and gait after lumbar surgery ${ }^{18,37,38,39,40}$, but it can also be applied for other surgical interventions of the musculoskeletal system.

The investigator has to be aware of some method-related pitfalls. The rasterstereographic analysis of the back profile is highly dependent on the precise selection of the anatomical landmarks. If chosen imprecisely, measurement and data calculation will be incorrect as well. In addition, the subject's back must be completely undressed. Even wires of a bra or long scalp hair could disturb the scanning process. As gait measurements are susceptible to limping as a result of a painful hip, knee or ankle joint, the tested subjects need to be well examined before inclusion in the study and also before each follow-up visit to ensure the results are relevant and in correlation to the alterations of the spine. Since both methods have a high intra- and interobserver reliability ${ }^{21,24,41,42}$, their use in every-day routine can be easily implemented. However, combining both measurement-techniques might make it difficult to keep track of the abundance of data and to interpret these findings in a justifiable time.

One limitation of the technique of back surface measurement in general is that, to date, the data in the literature mostly refer to radiologic parameters obtained from X-rays to interpret postoperative outcome ${ }^{24}$. Since - due to modality-specific limitations - the definition of parameters used to describe posture differs between rasterstereography and X-rays (for example thoracic angle: rasterstereography thoracic vertebrae 1 to 12 , x-ray thoracic vertebrae 4 to 12) it is not yet possible to derive conclusions from absolute values obtained by rasterstereographic analysis. It is rather their changes in the perioperative course that are of interest. Presently this tool is thus best suited for longitudinal analyses.

Other objectifiable data, such as CT (computed tomography imaging) or MRI (magnetic resonance imaging), can help to technically evaluate postoperative outcome, but they only illustrate static anatomical details. In contrast to the non-invasive and radiation-free measurement techniques described in this protocol, these imaging techniques are not able to take function into consideration ${ }^{8,9,10}$

Interestingly the changes for gait and posture in our study were not always related with the patients' levels of pain. It thus appears that the postoperative dimension of function is not strictly associated with pain experience. The observed functional results are thus to be considered not contradictory but rather complementary to the patient related outcome measures. These measurements hence offer an additional dimension to critically evaluate postoperative outcome.

The evaluation of gait and posture is still a highly dynamic research field. We are confident that providing data about perioperative development of such functional parameters will improve our understanding of these conditions. In the long run, this may also help to further improve our surgical outcomes. 
It is, therefore, important to apply the technique described in detail in this protocol and video on a broader scale to obtain more data about the functional parameters posture and gait in the perioperative course of musculoskeletal surgery.

\section{Disclosures}

The authors have nothing to disclose.

\section{Acknowledgments}

The authors have no acknowledgements.

\section{References}

1. Deyo, R. A., Nachemson, A., Mirza, S. K. Spinal-fusion surgery-the case for restraint. The Spine Journal. (2004).

2. Rajaee, S. S., Bae, H. W., Kanim, L. E. A., Delamarter, R. B. Spinal Fusion in the United States. Spine. 37 (1), $67-76$ (2012).

3. Faraj, S. S. A. et al. Measuring outcomes in adult spinal deformity surgery: a systematic review to identify current strengths, weaknesses and gaps in patient-reported outcome measures. European Spine Journal: official publication of the European Spine Society, the European Spinal Deformity Society, and the European Section of the Cervical Spine Research Society. 26 (8), 2084-2093 (2017).

4. Maughan, E. F., Lewis, J. S. Outcome measures in chronic low back pain. European Spine Journal: Official Publication of the European Spine Society, the European Spinal Deformity Society, and the European Section of the Cervical Spine Research Society. 19 (9), $1484-1494$ (2010).

5. Vavken, P. et al. Fundamentals of Clinical Outcomes Assessment for Spinal Disorders: Clinical Outcome Instruments and Applications. Global Spine Journal. 5 (4), 329-338 (2014).

6. Weishaupt, D., Zanetti, M., Boos, N., Hodler, J. MR imaging and CT in osteoarthritis of the lumbar facet joints. Skeletal Radiology. 28 (4), 215-219 (1999).

7. Pathria, M., Sartoris, D. J., Resnick, D. Osteoarthritis of the facet joints: accuracy of oblique radiographic assessment. Radiology. 164 (1), 227-230 (1987).

8. Ract, I. et al. A review of the value of MRI signs in low back pain. Diagnostic and Interventional Imaging. 96 (3), $239-249$ (2015).

9. Elfering, A. et al. Risk factors for lumbar disc degeneration: a 5-year prospective Mri study in asymptomatic individuals. Spine. 27 (2), 125-134 (2002).

10. Ashraf, A. et al. Correlation between Radiologic Sign of Lumbar Lordosis and Functional Status in Patients with Chronic Mechanical Low Back Pain. Asian spine journal. 8 (5), 565-570 (2014).

11. Glassman, S. D. et al. The impact of positive sagittal balance in adult spinal deformity. Spine. 30 (18), 2024-2029 (2005).

12. Glassman, S. D., Berven, S., Bridwell, K., Horton, W., Dimar, J. R. Correlation of radiographic parameters and clinical symptoms in adult scoliosis. Spine. 30 (6), 682-688 (2005).

13. Sangtarash, F., Manshadi, F. D., Sadeghi, A. The relationship of thoracic kyphosis to gait performance and quality of life in women with osteoporosis. - PubMed - NCBI. Osteoporosis International. 26 (8), 2203-2208 (2015).

14. Miyakoshi, N., Itoi, E., Kobayashi, M., Kodama, H. Impact of postural deformities and spinal mobility on quality of life in postmenopausal osteoporosis. Osteoporosis International. 14 (12), 1007-1012 (2003).

15. Imagama, S. et al. Back muscle strength and spinal mobility are predictors of quality of life in middle-aged and elderly males. European Spine Journal: Official Publication of the European Spine Society, the European Spinal Deformity Society, and the European Section of the Cervical Spine Research Society. 20 (6), 954-961 (2010).

16. Barrey, C. Current strategies for the restoration of adequate lordosis during lumbar fusion. World Journal of Orthopedics. 6 (1), 117-11 (2015).

17. Drerup, B. A procedure for the numerical analysis of moiré topograms. Photogrammetria. 36 (2), 41-49 (1981).

18. Drerup, B., Hierholzer, E. Automatic localization of anatomical landmarks on the back surface and construction of a body-fixed coordinate system. Journal of Biomechanics. 20 (10), 961-970 (1987).

19. Meadows, D. M., Johnson, W. O., Allen, J. B. Generation of surface contours by moiré patterns. - PubMed - NCBI. Applied Optics. 9 (4), 942-947 (1970).

20. Takasaki, H. Moiré Topography. Applied Optics. 9 (6), 1467-1472 (1970).

21. Schroeder, J., Reer, R., Braumann, K. M. Video raster stereography back shape reconstruction: a reliability study for sagittal, frontal, and transversal plane parameters. European Spine Journal: Official Publication of the European Spine Society, the European Spinal Deformity Society, and the European Section of the Cervical Spine Research Society. 24 (2), 262-269 (2015).

22. Frobin, W., Hierholzer, E. Transformation Of Irregularly Sampled Surface Data Points Into A Regular Grid And Aspects Of Surface Interpolation, Smoothing And Accuracy. 1985 International Technical Symposium/Europe. 0602, 109-115 (1986).

23. Hackenberg, L., Hierholzer, E., Pötzl, W., Götze, C., Liljenqvist, U. Rasterstereographic back shape analysis in idiopathic scoliosis after anterior correction and fusion. Clin Biomech. 18 (1), 1-8 (2003).

24. Mohokum, M., Schülein, S., Skwara, A. The validity of rasterstereography: a systematic review. Orthopedic Reviews. 7 (3), 1-6 (2015).

25. Tabard-Fougère, A. et al. Validity and Reliability of Spine Rasterstereography in Patients With Adolescent Idiopathic Scoliosis. Spine. 42 (2), 98-105 (2017).

26. Scheidt, S., Endreß, S., Gesicki, M., Hofmann, U. K. Using video rasterstereography and treadmill gait analysis as a tool for evaluating postoperative outcome after lumbar spinal fusion. Gait, Posture. 64, 18-24 (2018).

27. Lamoth, C. J. C., Daffertshofer, A., Meijer, O. G., Beek, P. J. How do persons with chronic low back pain speed up and slow down? Gait, Posture. 23 (2), 230-239 (2006).

28. Taylor, N. F., Evans, O. M., Goldie, P. A. The effect of walking faster on people with acute low back pain. European Spine Journal: Official Publication of the European Spine Society, the European Spinal Deformity Society, and the European Section of the Cervical Spine Research Society. 12 (2), 166-172 (2003). 
29. Bryant, A. R., Tinley, P., Cole, J. H. Plantar pressure and radiographic changes to the forefoot after the Austin bunionectomy. Journal of the American Podiatric Medical Association. 95 (4), 357-365 (2005).

30. Titianova, E. B., Mateev, P. S., Tarkka, I. M. Footprint analysis of gait using a pressure sensor system. - PubMed - NCBI. Journal of Electromyography and Kinesiology. 14 (2), 275-281 (2004).

31. Hennig, E. M., Milani, T. L. [The tripod support of the foot. An analysis of pressure distribution under static and dynamic loading]. Zeitschrift für Orthopädie und ihre Grenzgebiete. 131 (3), 279-284 (1993).

32. da Fonseca, J. L., Magini, M., de Freitas, T. H. Laboratory Gait Analysis in Patients with Low Back Pain before and after a Pilates Intervention. Journal of Sport Rehabilitation. 18 (2), 269-282 (2009)

33. Hayashi, K. et al. Gait Speeds Associated with Anxiety Responses to Pain in Osteoarthritis Patients. Pain medicine (Malden, Mass.). 17 (3), 606-613 (2016).

34. Fairbank, J. C. T., Pynsent, P. B. The Oswestry Disability Index. Spine. 25 (22), 2940 (2000).

35. Hawker, G. A., Mian, S., Kendzerska, T., French, M. Measures of adult pain: Visual Analog Scale for Pain (VAS Pain), Numeric Rating Scale for Pain (NRS Pain), McGill Pain Questionnaire (MPQ), Short-Form McGill Pain Questionnaire (SF-MPQ), Chronic Pain Grade Scale (CPGS), Short Form-36 Bodily Pain Scale (SF-36 BPS), and Measure of Intermittent and Constant Osteoarthritis Pain (ICOAP). Arthritis Care, Research. 63 (S11), S240-S252 (2011).

36. Haefeli, M., Elfering, A. Pain assessment. European Spine Journal: Official Publication of the European Spine Society, the European Spinal Deformity Society, and the European Section of the Cervical Spine Research Society. 15 (S1), S17-S24 (2005).

37. Drerup, B., Hierholzer, E. Evaluation of frontal radiographs of scoliotic spines--Part I. Measurement of position and orientation of vertebrae and assessment of clinical shape parameters. Journal of Biomechanics. 25 (11), 1357-1362 (1992).

38. Drerup, B., Hierholzer, E. Evaluation of frontal radiographs of scoliotic spines--Part II. Relations between lateral deviation, lateral tilt and axial rotation of vertebrae. Journal of Biomechanics. 25 (12), 1443-1450 (1992).

39. Drerup, B., Hierholzer, E. Back shape measurement using video rasterstereography and three-dimensional reconstruction of spinal shape. Clinical biomechanics (Bristol, Avon). 9 (1), 28-36 (1994).

40. Abdul Razak, A. H., Zayegh, A., Begg, R. K., Wahab, Y. Foot Plantar Pressure Measurement System: A Review. Sensors. 12 (7), $9884-9912$ (2012).

41. Melvin, M., Mohokum, M., et al. Reproducibility of rasterstereography for kyphotic and lordotic angles, trunk length, and trunk inclination: a reliability study. Spine. 35 (14), 1353-1358 (2010).

42. Liljenqvist, U., Halm, H., Hierholzer, E., Drerup, B., Weiland, M. Die dreidimensionale Oberflächenvermessung von Wirbelsäulendeformitäten anhand der Videorasterstereographie*. Zeitschrift für Orthopädie und ihre Grenzgebiete. 136 (01), 57-64 (1998). 